East African Medical Journal Vol. 77 No. 4 April 2000

POST-TRAUMATIC STRESS DISORDER: CASE REPORT

C. M. Nyamai, MBChB (Nbi), MSc Psych (UK)., African Air Rescue Health Services, Nairobi, P.O. Box 7106, Nairobi and F. G. Njenga, MBChB (Nbi), DPM (London), MRC Psych (UK), Consultant Psychiatrist, P.O. Box 73749, Nairobi.

\title{
POST-TRAUMATIC STRESS DISORDER: CASE REPORT
}

\author{
C. M. NYAMAI and F. G. NJENGA
}

\begin{abstract}
SUMMARY
Following exposure to a major traumatic event like the August seventh 1998 Nairobi bomb blast various reactions occur, some of which result in stress-related psychiatric disorders. We have described one such case, and used it to illustrate the salient features of posttraumatic stress disorder. We have outlined the diagnostic categories of the post-traumatic disorders and discussed the different treatment modalities applicable.
\end{abstract}

\section{INTRODUCTION}

Africa is a highly traumatised continent. Natural and man-made calamities abound. From South Africa to Egypt frequent reports are made of domestic violence, war related crimes against the person, famines, floods, and forced migrations as a result of natural and man-made disasters. Africa has the largest numbers of refugees and witnessed the most tragic act of genocide in Rwanda in 1994.

Common traumas do not often attract attention. Kenya has one of the highest rates of fatal road accidents per car in the world. Post-traumatic stress disorders are a common consequence of RTA's even in children and adolescents(1). Terrorism is an ever-present threat to the civilised world. No group of people is spared in a terrorist attack. The literature on the consequences of trauma in Africa is conspicuous by its paucity. No publication relative to an act of terrorism in Black Africa was found by the authors following an extensive literature search.

Studies have been published relative to the Oklahoma bomb disaster and the violence in northern Ireland. Community-based research on the impact of political violence on psychiatric morbidity in northern Ireland has shown that those who lived in a more violent town had higher scores on the General Health Questionnaire (GHQ) than those living in a less violent town, indicating poorer mental health(2).

Following the bombing in Enniskillen (northern Ireland) in 1987 in which eleven people were killed and 60 injured, all victims had high scores on the GHQ indicating poor mental health when examined at six months and at one year, and $50 \%$ of the survivors examined had developed PTSD at six months(3). The significance of this finding to the August seventh 1998 bomb blast in Nairobi where casualties were much higher are self evident and demand urgent systems of surveillance of the population.

This case report is intended to bring to the attention of the reader the symptoms of post-traumatic stress disorder and the different treatment modalities applicable. It is hoped that this case report will stimulate interest and discussion among psychiatrists and other mental health workers in the area of PTSD. In particular, it is hoped that systemic follow up of various groups of survivors will be the subject of scientific inquiry with the expectation that the tragic event will avail science an opportunity for advancement.

\section{CASE REPORT}

$\mathrm{M}$ is a 38 year old married man with three children, and works as a lift technician. He presented to the psychiatric clinic six weeks after the bomb blast following referral by his general practitioner (GP).

He had previously been well, and was repairing a lift in the machine room above the twenty first floor of a building adjacent to the American Embassy in Nairobi when the bomb went off on August seventh 1998.

Just on the moment of the first blast (grenade) he had flipped on the repaired lift's electrical switch, giving him the initial impression that he had caused the blast through an electrical fault.

His first concern was that he had caused the death of his two colleagues. He flipped the switch off, the major blast went off (one ton of TNT). The place went dark with dust all over, and he was flung to the floor, and knew that he, too, was going to die.

Moments later he heard one of his colleagues call his name, and was relieved to learn that they were still alive. They made their way down the staircase, now heavily blood stained and smelling of blood, amid all the screaming and confusion, stepping over dead bodies, and turning into rescuers on the way as they helped carry injured people down the narrow stairway.

After finally getting out of the building, $M$. remembered he had left the lifts' switch off. Not able to bear the thought of people trapped in the lifts because of this, he went back into the building amid protests from those around for his safety. He went up all the floors to confirm that the lifts were open and that no one was trapped inside. It was while looking down the shaft to ascertain that no one had been trapped there that he sustained glass cut wounds on his hands. He had otherwise not been physically injured.

He finally came out of the building again, and just stood outside transfixed, staring at the rescue process for five hours. It was then that it finally occurred to him that his family would be 
worried about him, and he went home. He has no recollection of his thoughts as he watched the rescue operation, other than that he was "shocked".

That night he could not sleep. He kept seeing images of the blast scene, the injured, bleeding people, the bodies they were stepping over. He developed an aversion to meat and did not want to see anyone eating it, let alone eat it himself. His family had to prepare and take it elsewhere. He became very sensitive to noise, closing doors would get on his nerves. He wanted to keep quietly to himself. This continued for three days then he felt somewhat better and was able to go back to work.

He tried to use his work to distract himself from what he had gone through, but as workmates and friends kept asking him about that fateful day this proved rather difficult. He now started actively avoiding such people in order to avoid talking about what he had experienced.

One week later he developed some body aches and pains. He saw his GP and was started on analgesics. These did not give him any relief. He had further difficulties with sleep, loss of appetite, started experiencing palpitations, had an uncomfortable sensation in his stomach, a sense of fear, and a feeling that something bad was going to happen (foreboding). He was constantly worried, his mood became low, he developed feelings of guilt, wondering why he had survived when so many people had died in the blast (acute stress reaction - DMS IV)(4).

He became afraid of being alone. He found it difficult to communicate with those around him including his own family members. He found that he had lost all sexual interest, and could not achieve an erection. It was at this point that he again saw his GP, who referred him for psychiatric help. Here he was evaluated and debriefing done. He was started on a hypnotic and an SSRI to help with his symptoms. Psychotherapy was started to help him synthesise the experience he had gone through, and to help him understand what exactly was happening to him. Cognitive and behavioural techniques were used as the mainstay of his therapy, and sildenafil (viagra) prescribed for the sexual dysfunction with very good results. He has now regained his sexual function, which has helped to restore his self-image and self esteem.

\section{DISCUSSION}

$M$ is a typical example of the effects that follow a traumatic experience of this nature. He suffered the acute stress disorder as well as post traumatic stress disorder (PTSD).

Following exposure to the trauma of a disaster, psychological morbidity has been reported to affect some $30-40 \%$ of the affected population within the following first year(5). Disasters that are man-made and with shock and destruction have shown persistent levels of over 30\% severe impairment(5).

The symptoms this patient presented with are typical of post-traumatic stress symptoms, which can be divided into three major categories (DSM IV classification)(4): persistent re-experiencing of the event - the intrusive symptoms. These include the nightmares, vivid recollections/flashbacks, a feeling of re-living the episode; Persistent avoidance of any stimuli that remind one of the trauma and numbing of general responsiveness - the building, going into town, work-mates and; A hyperarousal state leading to hypervigilance, startle reflexes, sleep disturbance.
All these lead to some degree of psychosocial impairment with affected people unable to continue with activities of daily living due to the symptoms. Other symptoms include emotional liability, irritability, restlessness, and sometimes outbursts of violent behaviour. Substance abuse, particularly of alcohol, may follow attempts to self medicate.

Depending on the time of onset and the duration of symptoms relative to the traumatic event, the resulting disorders can be categorised according to the DSM IV 4 into: acute stress reaction - symptoms occur within four weeks of the traumatic event and last between two days and four weeks; acute PTSD - symptoms lasting less than three months; Chronic PTSD - symptoms lasting more than three months and; Delayed onset PTSD - onset of symptoms is at least six months after the stressor.

Useful predictors of outcome can be divided into those that are event-related, those related to the individual, and to the community. In the event-related factors, poorer prognosis will be related to a trauma that is sudden, unexpected, causes a lot of loss of life, is man-made, and has the likelihood of recurring. Individual vulnerability will be related to proximity to the disaster, severity of physical injury, exposure to multiple deaths and mutilation, concurrent life stresses, perceived threat to life, being trapped, and absence of local social and family supports(6).

Treatment: The goal is to relieve the suffering experienced, and to prevent progression to the more chronic, more debilitating, and more difficult to treat PTSD, and to foster resilience(7). This consists of debriefing and counselling, which should be done as early as possible after the traumatic event. Debriefing has been used as a stress management technique for groups exposed to traumatic events by several rescue organisations(8), its goal has been seen as primarily preventive, to help the victims deal with the inevitable stress so that problems do not arise subsequently.

Debriefing is done in groups and the major elements of trauma are reviewed by the participants. In the debriefing session the participants recount their respective experiences, what happened to them, what they saw, heard, touched, smelt, tasted, how they felt, the degree of perceived threat to life, what they thought, how they got away, and what has happened since(9).

Being able to do this helps the person get it off the chest, providing emotional ventilation and release, and helps the cognitive processing of the information(8). In a group setting, hearing that others experienced similar fear, or also cried, reassures one of his normality in the circumstances.

Evidence of the effectiveness of debriefing is scanty as most is anecdotal with follow up data lacking(8). Some studies have shown that it is useful(8), and others that it is doubtful(7). Our experience with the people we have worked with following this bomb blast is that it helps them feel better. The process of being listened to unconditionally and non-judgementally by an empathic listener could in 
itself contribute to this. Studies are on-going to establish the utility of these and other interventions.

Psycho-education involves teaching people what to expect after traumatic events(10). On an individual basis it involves an explanation of the basis of symptoms, the relationship between the trauma, the physiological, psychological and physical state This helps one understand better what is happening to them, and recognise symptoms for what they are rather than think of them as an additional "illness". On a community basis it involves educating the community on what to expect following the disaster; what is a normal reaction to an abnormal situation, and when to seek help. Psycho-education has demonstrated powerful efficacy after disaster events(10).

Supportive counselling aims at providing emotional and psychological support through this difficult time. A problem solving approach helps victims deal with issues arising from day to day living in their current state, the interpersonal and social problems related to PTSD. Relaxation techniques are useful particularly in the hyperarousal state.

Cognitive and behavioural techniques are very useful in dealing with the fear and the concomitant anxiety and depressive symptoms. They involve cognitive appraisal of the event -conscious, logical examination of the fear, its cause, and the thinking processes that maintain it, and seek to challenge negative automatic thoughts with rational reasoning. Gradual exposure to the feared stimuli help overcome the fear and the avoidance that may go with it.

Behavioural individual and group psychotherapy have been shown to be the best help for the negative symptoms of phobic avoidance and withdrawal associated with chronic PTSD

Co-morbidity: Assessment following acute trauma includes taking note of any pre-existing ailments, and recognition of co-existing ones. Depression, anxiety, phobic disorder, somatisation, and substance abuse are of particular note as they frequently occur, and need to be borne in mind during treatment(12).

PTSD patients are also at higher than normal risk for medical illness. Physiologically they exhibit abnormal cardiovascular function, endocrine dysregulation and immunosuppression(10).

Drug treatment: Adjunct treatment using the tricyclic antidepressants and the SSRI's is useful. Amitryptiline, imipramine, phenelzine and fluoxetine have demonstrated positive drug effects in PTSD relative to placebo(11). MAOI's are also useful, but their side effect profile limits their use.

The SSRI's have achieved impressive results with the avoidance symptoms(11) but do not affect abnormalities like the startle response(10). The TCA's and MAOI's have been shown to significantly reduce specific intrusive and hyperarousal anxiety symptoms of PTSD, but have had less effect on the avoidance symptoms(11). The impulse control problem has been shown to improve with the use of carbamazepine and lithium(11). This therefore explains the use, at times, of multidrug therapy.

Appropriate drug treatment of co-existing conditions is done in the usual manner. In this case use of sildenafil (viagra) for the sexual dysfunction proved very useful. In conclusion, this case report illustrates the symptoms that may occur following a major trauma. We have discussed the management of such conditions and the authors' interaction following the Nairobi bomb blast of August 1998. It is here suggested that the general principles of management of PTSD are applicable across cultures. The authors are following a group of trauma victims who will form the main part of the study now under way. This single case study is intended to draw the attention of the readers to the salient features of PTSD.

\section{REFERENCES}

1. Mirza K.A., Bhadrinath B.R., Goodyer I.M. and Gilmour C. Posttraumatic stress disorder in children and adolescents following road traffic accidents. Brit. J. Psychiat. 1998; 172:443-7

2. Cairns E. and Wilson R. The impact of political violence on mild psychiatric morbidity in Northern Ireland. Brit. J. Psychiat. 1984; 145:631-5.

3. Curran P.S., Bell P., Murray A., Loughrey G., Roddy R. and Rocke L.G. Psychological consequences of the Enniskillen bombing. Brit. J. Psychiat. 1990; 156:479-82.

4. American Psychiatric Association. Diagnostic and Statistical Manual of Mental Disorders 4th Edition Washington, DC 1994.

5. WHO Division of Mental Health, Psychosocial consequences of Disasters. Prevention and Management. Geneva 1992.

6. Alexander D.A. Psychological aspects of trauma. In: Key Topics in Trauma, Eds. Greaves A, Porter K, and Burke D. Bios Scientific Publishers Ltd. Oxford 1997.

7. Ursano R.J., Fullerton C.S. and Norwood A.E. Psychiatric dimensions of disaster: Patient Care, Community Consultation, and Preventive Medicine. In: Readings in Psychiatric Training for Disaster. American Psychiatric Association 1998 Annual meeting Toronto, Canada.

8. Shalev A.Y. Debriefing following traumatic exposure. Individual and Community responses to trauma and disaster: the structure of Human Chaos. Eds. R.J. Ursano, B.G. McCoughey and C.S. Fullerton. London: Cambridge University press, 1994.

9. Mitchel J.T.; When disaster strikes: The critical incident stress Debriefing process. J. Emerg. Med. Serv. 1983a; 8: 36-39.

10. Friedman M.J. Post-traumatic Stress Disorder. J. Clin. Psychiat. 1997; 58 (Suppl 9).

11. Wong J.C.M. and Ursano R.J. Traumatic Stress - An overview of Diagnostic Evolution and Clinical Treatment. Ann. Acad. Med. Singapore 1997; 26:76-78.

12. Brady K.T., 1997. Post Traumatic Stress Disorder and Co-morbidity: recognising the many faces of PTSD. J. Clin. Psychiat. 1997; 58 (suppl 9) pg 12-15. 